\title{
Two Cases of Malleostapedotomy in Congenital Oval Window Atresia
}

\author{
Sang Hyeon Ahn, Da Hee Kim, Jae Young Choi and Bo Gyung Kim \\ Department of Otorhinolaryngology, Yonsei University College of Medicine, Seoul, Korea
}

Received October 21, 2013

Revised November 19, 2013

Accepted November 27, 2013

\author{
Address for correspondence \\ Bo Gyung Kim, MD \\ Department of Otorhinolaryngology, \\ Yonsei University \\ College of Medicine, \\ 50 Yonsei-ro, Seodaemun-gu, \\ Seoul 120-752, Korea \\ Tel $+82-2-2228-3624$ \\ Fax +82-2-393-0580 \\ E-mail ally1118@yuhs.ac
}

Congenital anomaly of the oval window with an abnormal facial nerve course is an uncommon embryological defect, which is related to the underdevelopment of second branchial arch derivatives. Some treatments for improving hearing levels are available; these include hearing aids, vestibulotomy, neo-oval window formation, and stapes surgeries, including incudostapedotomy and malleostapedotomy. However, surgery for congenital anomalies of the oval window has rarely been described, usually in very small series of patients. We describe two cases of congenital anomalies of the oval window with aberrant facial nerve courses. One was a 40-yearold male diagnosed with unilateral congenital oval window atresia; the other was a 10-year-old male diagnosed with bilateral congenital oval window atresia. We also describe the clinical manifestations and treatment outcomes of malleostapedotomy for congenital anomalies of the oval window with aberrant facial nerve courses.

Korean J Audiol 2013;17:152-155

\section{Introduction}

Congenital anomalies of the oval window are due to malformations of the second branchial arch, in which there is not a full connection between the stapes and otic capsule, thereby preventing the oval window from being formed normally. ${ }^{1)}$ Congenital anomalies of the oval window have been reported in $0.5-1.2 \%$ of children with conductive hearing loss. ${ }^{2)}$ Various treatments for this anomaly have been introduced: hearing aids, ${ }^{3,4)}$ vestibulotomy, ${ }^{5)}$ fenestration of the horizontal semicircular canal, ${ }^{6,7)}$ and formation of a neo-oval window. ${ }^{8)}$ Also, as Fisch ${ }^{9)}$ reported, incudostapedotomy and malleostapedotomy have been used successfully to treat patients with congenital oval window anomalies. ${ }^{10)}$ However, in patients with accompanying malformations of the ossicles or abnormal routes of the facial nerve, the hearing improvement with previous procedures has not been sufficient. In our institute, instead of incudostapedotomy, we have performed malleostapedotomy, a safe and effective procedure for patients with oval

This is an Open Access article distributed under the terms of the Creative Commons Attribution Non-Commercial License (http://creativecommons org/licenses/by-nc/3.0/) which permits unrestricted non-commercial use, distribution, and reproduction in any medium, provided the original work is properly cited. window anomalies with accompanying ossicular malformations or facial nerve abnormalities. Here, we report two cases of malleostapedotomy performed in patients with hearing loss due to congenital anomalies of the oval window.

\section{Case Report}

\section{Patient 1}

A 40-year-old male visited our outpatient clinic with rightsided hearing loss since childhood. The patient had no previous history of trauma, and showed conductive hearing loss with an air-bone gap (ABG) of $50 \mathrm{~dB}$ (60/10 dB; Fig. 1A). Temporal bone computed tomography (TBCT) showed normal images of the malleus, but revealed atresia of the right-side oval window, with abnormal placement of the facial nerve (Fig. 1B). First, ossiculoplasty using a total ossicular replacement prosthesis (TORP) was performed, with no hearing improvement. We then planned a second operation, a malleostapedotomy. The tympanic cavity was exposed through an endaural approach and the posterior wall of the external auditory canal was leveled down with a mallet and gauge to expose the stapes. During the operation, a normal structure of the malleus was noted, but the long process of the incus was short. Addition- 
ally, the stapes was missing, and the facial nerve was covering the oval window. The facial nerve was displaced towards the cephalic side using a fine swab, and a 0.6- $\mathrm{mm}$ window was drilled in the obstructed oval window using a 0.4-mm Skeeter drill. The facial nerve was then put back in the original position. Because the malleus was placed anterior to the stapes footplate, the piston wire prosthesis was bent $20^{\circ}$, modified to a 7-mm length, and fixed on the normal malleus (Fig. 1C). At 1 year following the operation, pure tone audiometry showed improvement of the $\mathrm{ABG}$, from 50 to $30 \mathrm{~dB}$ (Fig. 1D), with no complication of facial nerve palsy or dizziness.

\section{Patient 2}

A 10-year-old male visited our outpatient clinic with bilateral hearing loss for several years. Audiometric evaluation showed bilateral conductive hearing loss (Fig. 2A), and TBCT revealed bilateral atresia of the oval window, with an abnormal array of the facial nerve (Fig. 2B). Explo-tympanoplasty was

Fig. 1. A: Preoperative pure-tone audiogram (PTA) of patient 1 showing conductive hearing loss in the right ear. B: Temporal bone CT imaging of patient 1 , showing oval window atresia and dislocation of the facial nerve (arrow). The malleus handle (head of arrow) appeared normal, but no stapedial structure was seen. C: Operative findings of patient 1 showed an abnormal incus with erosion and the facial nerve (FN) overhanging the oval window. A malleostapedotomy was conducted, bending a piston wire between the malleus handle and stapes footplate. D: Postoperative PTA of patient 1 showing improvement in right-side hearing.

Fig. 2. A: Preoperative PTA of patient 2 , showing conductive hearing loss in both ears. B: Temporal bone CT imaging of patient 2 . The head of the arrow indicates a normal malleus structure and the arrow shows oval window atresia with an aberrant course of the facial nerve. C: Operative findings of patient 2 , showing an eroded incus and the aberrant FN covering the oval window. D: Postoperative PTA of patient 2, showing improved hearing with a decreased air bone gap compared with the previous PTA. PTA: pure-tone audiogram, FN: facial nerve.
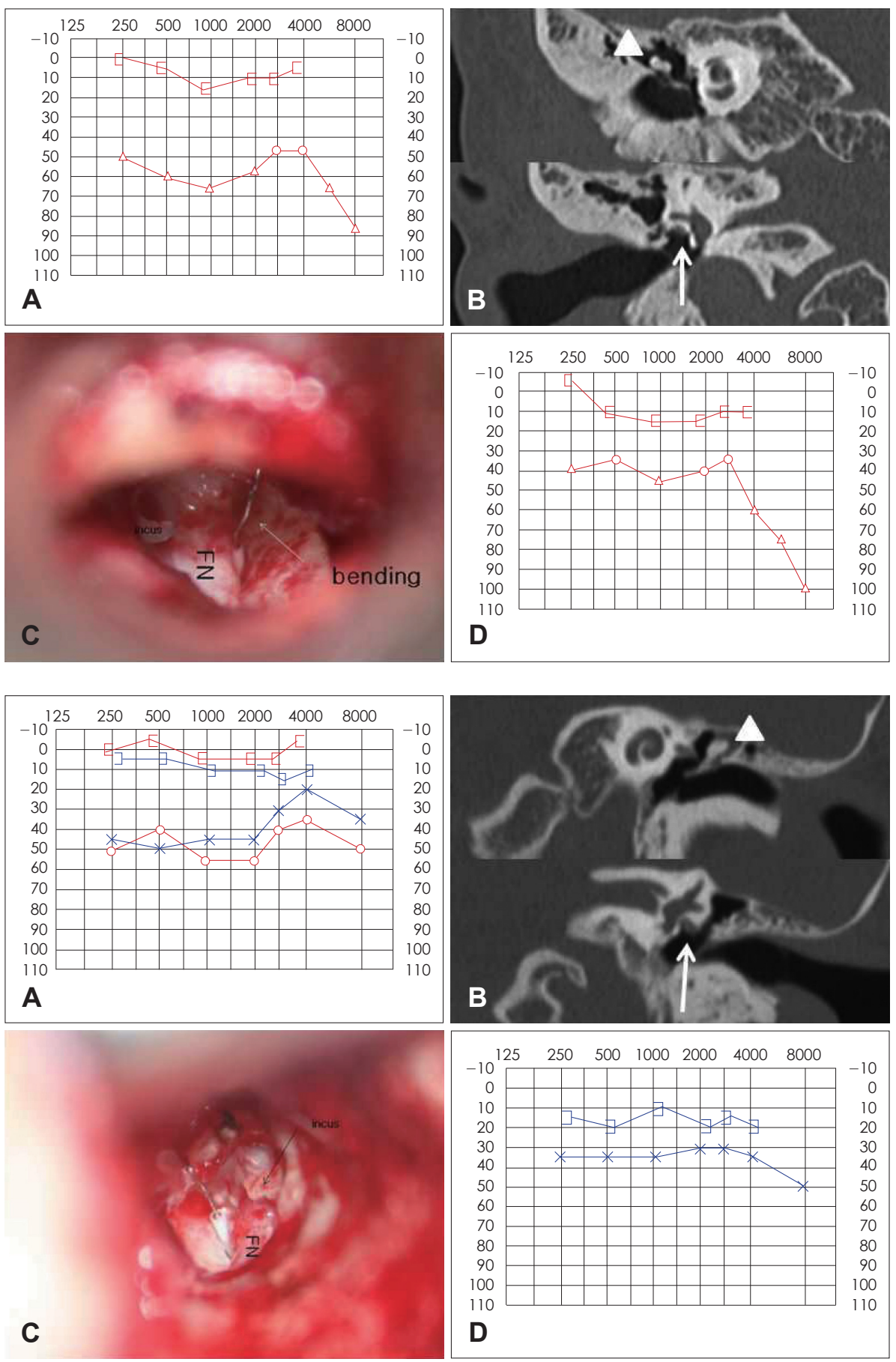
performed primarily on the left ear with an ossiculoplasty using a TORP; there was no hearing improvement. A stapedotomy was planned using a retroauricular approach. During the operation, the malleus showed normal images, but the long process of the incus was short. In addition, the stapes were missing, and the facial nerve was lying over the obstructed oval window. The facial nerve was cephalically rotated using the "facial roll" method, and a 0.6-mm window was created at the center of the stapes footplate using a $0.4-\mathrm{mm}$ Skeeter drill. A 7-mm-long piston wire was fixed on the malleus and tightened with piston forceps (Fig. 2C). Immediately postoperatively, the patient showed no dizziness or loss of taste, but mild facial palsy, House-Brackmann Grade III, was noted. By 1 month later, the facial palsy had recovered, to Grade I, and the $\mathrm{ABG}$ was reduced, from $40 \mathrm{~dB}$ (pre-operative) to $15 \mathrm{~dB}$ (6 month post-operative) on pure tone audiometry (Fig. 2D).

\section{Discussion}

Congenital anomalies of the oval window are rare; they are found in $0.5-1.2 \%$ of children with conductive hearing loss. ${ }^{1,2)}$ Cremer classified this congenital anomaly into four groups according to the abnormal structure of the middle ear. ${ }^{8}$ Type I was defined as fixation of the stapes footplate alone, and type II with stapes footplate fixation accompanied by other ossicular anomalies. Mobile stapes footplates with anomalies of other ossicles were classified as type III, aplasia of the oval or round window as type IVa, and an anomaly with dysplasia as type IVb. Here, patient 1 had a unilateral anomaly of type IVa, and patient 2 was also type IVa, but bilateral. Both cases had abnormal positioning of the facial nerve.

Several methods have been suggested to treat patients with congenital anomalies of the oval window and stapes. First, conservative treatment with hearing aids was proposed. Herman used bone-anchored hearing aids in five patients with aplasia of the oval window, ${ }^{3)}$ and Reiber and Schwaber ${ }^{4)}$ proposed using bilateral hearing aids in patients with ossicular anomalies, preserving the facial nerve.

Several surgical treatments, such as vestibulotomy, fenestration of the horizontal semicircular canal, and formation of a neo-oval window, were introduced subsequently. Lambert ${ }^{5)}$ performed vestibulotomies in six patients with congenital anomalies of the oval window by making a hole in the oval window and reconstructing the ossicles. Hearing improvement was shown in four patients, with 20-40-dB differences. Yi, et al. ${ }^{7)}$ reported two cases with improved hearing after perforating the horizontal semicircular canal in patients with aplasia of the stapes and the oval window. Teunissen and Cremers ${ }^{8)}$ reported 14 cases with aplasia of the oval window: a neo-oval window was formed in four patients, and Teflon inserts were placed with successful results in two patients. de Alarcon, et al. ${ }^{11)}$ described 17 patients; of them, 9 of 17 showed hearing improvement after neo-oval window formation and ossiculoplasties.

Finally, stapes surgery has been performed in cases of congenital anomalies of the oval window. Thomeer, et al. ${ }^{10)}$ performed surgery on 15 ears with congenital anomalies. Three patients underwent incudostapedotomies, and only one (33\%) showed an ABG improvement, of $20 \mathrm{~dB}$. Two patients underwent malleostapedotomies, with a $50 \%$ success rate. In one patient, the ABG improved, with a 46-dB difference, but in the other patient, the footplate of the stapes was obstructed and the malleus was broken.

Stapedotomies, including incudostapedotomy and malleostapedotomy, are usually performed in otosclerosis patients and congenital ossicular anomaly patients. Malleostapedotomy involves connecting the malleus directly to the vestibule through the oval window, instead of connecting the incus and the stapes. This operation is used in cases where an incudostapedotomy is not possible because the malleus head or the incus is missing or fixed. It can also be used in cases that need a second operation after an ossiculoplasty or where the lenticular process is necrotized after an incudostapedotomy. ${ }^{12)}$

In $90 \%$ of stapedotomy cases, the facial nerve is exposed with no problem, but in a few cases the facial nerve covers the oval window, blocking the approach to the stapes footplate. In these cases, the "facial roll" method, rolling the facial nerve gently upwards, can be used to ease the approach to the oval window. Even if the facial nerve is abutting the piston wire prosthesis, it is known to cause no facial nerve or hearing dysfunction. ${ }^{13)}$ In both cases in this report, we used the facial roll method to expose the oval window; mild facial palsy was noted in the second case, but recovered fully. This was thought to have been caused transiently by nerve traction. If attention is paid to minimizing nerve traction in this procedure, good exposure can be achieved without facial palsy.

There has been no previous report in Korea of the results of the malleostapedotomy technique in patients with congenital oval window anomalies and accompanying ossicular malformations and abnormal routes of the facial nerve. The follow-up period after the surgery was short in these two cases, and longer-term follow-up assessments are necessary. However, through this easy and safe surgical method, hearing improved in a short period of time. Malleostapedotomy has the potential to replace previous procedures and is safe and effective in patients with congenital oval window anomalies. 


\section{REFERENCES}

1) Harada T, Black FO, Sando I, Singleton GT. Temporal bone histopathologic findings in congenital anomalies of the oval window. Otolaryngol Head Neck Surg (1979) 1980;88:275-87.

2) Briggs RJ, Luxford WM. Correction of conductive hearing loss in children. Otolaryngol Clin North Am 1994;27:607-20.

3) Herman HK, Kimmelman CP. Congenital anomalies limited to the middle ear. Otolaryngol Head Neck Surg 1992;106:285-7.

4) Reiber ME, Schwaber MK. Congenital absence of stapes and facial nerve dehiscence. Otolaryngol Head Neck Surg 1997;116:278.

5) Lambert PR. Congenital absence of the oval window. Laryngoscope 1990;100:37-40.

6) Shambaugh GE Jr. The fenestration operation for otosclerosis. Minn Med 1947;30:1249.

7) Yi Z, Yang J, Li Z, Zhou A, Lin Y. Bilateral congenital absence of stapes and oval window in 2 members of a family: etiology and management. Otolaryngol Head Neck Surg 2003;128:777-82.
8) Teunissen EB, Cremers WR. Classification of congenital middle ear anomalies. Report on 144 ears. Ann Otol Rhinol Laryngol 1993;102 (8 Pt 1):606-12.

9) Fisch U. Tympanoplasty, Mastoidectomy, and Stapes surgery. New York: Thieme;1994.

10) Thomeer H, Kunst H, Verbist B, Cremers C. Congenital oval or round window anomaly with or without abnormal facial nerve course: surgical results for 15 ears. Otol Neurotol 2012;33:779-84.

11) de Alarcon A, Jahrsdoerfer RA, Kesser BW. Congenital absence of the oval window: diagnosis, surgery, and audiometric outcomes. Otol Neurotol 2008;29:23-8.

12) Fisch U, Acar GO, Huber AM. Malleostapedotomy in revision surgery for otosclerosis. Otol Neurotol 2001;22:776-85.

13) Sterkers JM, Sterkers O. Surgical management of congenital absence of the oval window with malposition of the facial nerve. Adv Otorhinolaryngol 1988;40:33-7. 